\title{
Extreme wave-structure interaction and directional spreading effects on the air-gap design of an offshore platform
}

\author{
M. Chen \\ Department of Engineering, University of Cambridge, UK
}

\begin{abstract}
Accurate prediction on the behaviour of the largest waves in an ocean environment is vital to the safe design and performance of offshore platforms. This paper is concerned with the description of extreme waves, and includes both linear and nonlinear modelling of the largest water waves arising within directionally spread sea-state. The statistical description of the most probable temporal shape of the free surface in the vicinity of a large crest, for a given wave period and wave direction is found to correspond to the statistics of an extreme wave event. A variety of time-frequency analysis techniques is used to demonstrate that the free surface elevation time histories of focused wave groups are in close agreement with linear theory. A deterministic nonlinear correction is proposed for isolated extreme storm events. The NewWave formulation associated with this proposed nonlinear correction coefficient is employed to simulate the interactions between an extreme wave event and an idealized platform loading column. Statistics of the predicted maximum crest elevation are obtained using an exact linear interaction formulation of the scattering of water waves by a vertical circular cylinder in conjunction with a unidirectional Bretschneider spectrum. The results show that the second-order coefficient of the Stokes-type wave component is affected by directional spreading. Statistical analyses suggest that second-order wave theory describes the wave field very accurately even for extreme events. The present study provides a critical insight into the free surface elevation maxima of random seas containing waves of different frequencies and various energies, thus safeguarding the air-gap design of the offshore platform.
\end{abstract}

Keywords: extreme wave, wave-structure interaction, air-gap design, nonlinear modelling, directional spreading. 


\section{Introduction}

The rapid growth of ocean transportation and exploration for energy industries and national defenses has emphasised a need for predicting accurate extreme wave effects for both safety and economic reasons, such as offshore operations including offshore tank associated with its mooring, offshore terminals, oil platforms associated with drilling rigs and coastal protections. In particular, the context of water wave diffraction and radiation caused by man-made coastal and offshore structures or boundary conditions is of considerable significance to these issues. In the past, many empirical distribution functions have been proposed for wave crest heights. But the resulting predictions either overestimate or underestimate the crest heights. A primary reason for this is the difficulty of obtaining the true local height of the wave crests from measurements. Also, the location of instruments plays an important role. It is well known that steep water waves are nonlinear. In general, the non-linearity is weak, but it is vital that the nonlinearity is taken into account in order to record a more accurate crest height. From a practical standpoint, nonlinear effects are crucial when assessing forces on offshore structures, wave set-up at coasts, mooring forces, and harbour surging. In an effort to simplify the interaction theory, Mei [3] examined the water wave diffraction and radiation extensively using the linearised theory and some analytical solutions proposed by Ursell [4].

For the incompressible and irrotational flow of free surface tension effects to be neglected, the foremost nonlinear contribution to the wave motions can be properly estimated to second order. This paper focuses on the accurate secondorder analytical solution for nonlinear inviscid waves and typical wave phenomena revealed by this solution. The mathematical representation of the nonlinearity of a random sea state mostly arises through the advection terms in the basic hydrodynamic equation (i.e. $u \partial u / \partial x$, where $u$ is the horizontal component of water particle velocity). The advantage of using second order wave theory is that the effects of wave steepness, directional spreading information and water depth are included without any approximation. However, it is worthy to reiterate that the analysis of wave-structure interaction is a nonlinear, three dimensional problem, which has not yet been fully understood. In particular, the disturbance to the nonlinear free surface motion due to wave diffraction around vertical circular cylinder arrays is of great relevance to this problem. It was Longuet-Higgins [5] who first derived a second-order theory for deep water wave-wave interactions. Later, Lindgren [6] developed a model for extremes within a random sea-state in a purely mathematical context. Using an approach similar to Lindgren [6], Boccotti [7] found that the average shape of a large crest in both time and space is a scaled version of the autocorrelation function. Linton and Evans [8] applied the interaction theory to linear arrays of cylinders among incident NewWaves. Tromans and Anaturk [9] provided a full derivation of NewWave theory, in the context of offshore engineering. Taylor and Jonathan [10] compared predictions based on the NewWave model with field data from the North Sea, and concluded that NewWave effectively models the expected shape of the largest waves. Forristall [11] suggested a correction to 
linear wave theory that incorporated second-order nonlinearity, and showed that the predictions were in close agreement with measured data for a set of JONSWAP spectra.

In reality, waves in the ocean do not necessarily propagate in the same direction as the local wind (i.e. the spectrum must consist of energy spreading in various directions). The directionality of the wave field depends on the distribution of energy in the directional domain. In addition, uncertainties still arise from the measured field and instrument action (Forristall [11]). Therefore, the effect of directional spreading on the free surface elevation will be investigated by a spectrum of known properties in this paper. In turn, this investigation commences from a focused linear wave group and the second-order sum components for a given Bretschneider spectrum with known properties, followed by full nonlinear computation of directional wave components associated with a random phase. Both shape and magnitude of the free surface elevation will be discussed and compared. Conclusions are drawn alongside numerical predictions.

\section{Directional spreading effects and wave-wave interaction}

In order to obtain directional second-order wave-wave interaction coefficients, it is sensible to consider the situation with two linear, superimposed monochromatic wave components. Although, this is a considerable simplification of general second-order wave-wave interactions, it does provide insight into certain important characteristics of the hydrodynamics of irregular waves. In order to represent a second-order wave-wave interaction solution, two linear wave components are required. Further details of mathematical derivation of Stokes water wave theory and formulation of interaction theory are given by Dean and Dalrymple [1] and Linton and Evans [8]. The resulting second-order expressions are:

$$
\Phi^{(2)}+g \Phi_{z}^{(2)}=\frac{-\partial}{\partial t}\left|\mathbf{u}^{(1)}\right|^{2} \text { at } z=0
$$

and

$$
\eta^{(2)}=\frac{-1}{g}\left[\frac{1}{2}\left|\mathbf{u}^{(1)}\right|^{2}+\Phi_{t}^{(2)}+\eta^{(1)} \Phi_{t z}^{(1)}\right] \text { at } z=0 .
$$

The choice of wave amplitude, wave frequency, and wave direction, for the two linear wave components is arbitrary. Evaluating the right hand side of eqn. (1):

$$
\frac{-\partial}{\partial t}\left|\mathbf{u}^{(1)}\right|^{2}=-a_{1} a_{2} \omega_{1} \omega_{2}\left[\begin{array}{l}
\left(1+\gamma_{12}\right)\left(\omega_{1}-\omega_{2}\right) \sin \left(\psi_{1}-\psi_{2}\right)- \\
\left(1-\gamma_{12}\right)\left(\omega_{1}+\omega_{2}\right) \sin \left(\psi_{1}+\psi_{2}\right)
\end{array}\right]
$$


where $\gamma_{12}=\cos \left(\theta_{1}-\theta_{2}\right) . \theta_{1}$ and $\theta_{2}$ are the propagation directions of $k_{1}$ and $k_{2}$.

Assuming a solution to $\Phi^{(2)}$ which obeys both Laplace's equation and the bed boundary condition:

$$
\Phi^{(2)}=B_{1} \sin \left(\psi_{1}-\psi_{2}\right) e^{k_{12} Z}+B_{2} \sin \left(\psi_{1}+\psi_{2}\right) e^{k_{12}^{+} Z}
$$

where the $B_{1}$ and $B_{2}$ coefficients are yet-to-be-determined functions of the firstorder wave numbers and frequencies, and $k_{12}^{+}$and $k_{12}^{-}$are the magnitudes of the respective wave number vectors (see Figure 1 for a definition sketch).

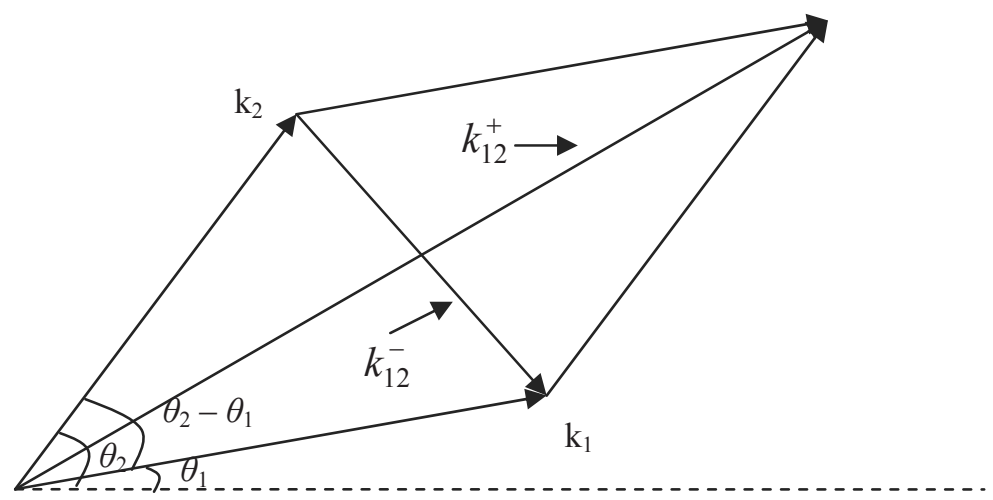

Figure 1: Diagram to illustrate the relationship between $k_{12}^{+}, k_{12}^{-}$and directional spreading angle $\theta=\theta_{1}-\theta_{2}$.

Similarly, the vector sum and vector difference between the wave numbers:

$$
k_{12}^{+}=\left(k_{1}^{2}+k_{2}^{2}+2 k_{1} k_{2} \gamma_{12}\right)^{1 / 2}
$$

and

$$
k_{12}^{-}=\left(k_{1}^{2}+k_{2}^{2}-2 k_{1} k_{2} \gamma_{12}\right)^{1 / 2}
$$

where the wave number vector of the $n$th components,

$$
\mathbf{k}_{n}=\left(k_{x}, k_{y}\right)=\left(k_{n} \cos \theta_{n}, k_{n} \sin \theta_{n}\right) \text {, in which } n=1,2 .
$$

After differencing eqn. (4) and substituting into the left hand side of eqn. (1),

$$
\Phi^{(2)}+\left.g \Phi_{z}^{(2)}\right|_{z=0}=-\left[\begin{array}{l}
B_{1}\left(\left(\omega_{1}-\omega_{2}\right)^{2}-g k_{12}^{-}\right) \sin \left(\psi_{1}-\psi_{2}\right)+ \\
B_{2}\left(\left(\omega_{1}+\omega_{2}\right)^{2}-g k_{12}^{+}\right) \sin \left(\psi_{1}+\psi_{2}\right)
\end{array}\right]
$$


Substituting eqn. (8) into eqn. (3), the coefficients $B_{1}$ and $B_{2}$ are given by:

$$
B_{1}=a_{1} a_{2} \omega_{1} \omega_{2} \frac{\left[\left(1+\gamma_{12}\right)\left(\omega_{1}-\omega_{2}\right)\right]}{\left(\omega_{1}-\omega_{2}\right)^{2}-g k_{12}^{-}},
$$

and

$$
B_{2}=-a_{1} a_{2} \omega_{1} \omega_{2} \frac{\left[\left(1-\gamma_{12}\right)\left(\omega_{1}+\omega_{2}\right)\right]}{\left(\omega_{1}+\omega_{2}\right)^{2}-g k_{12}^{+}} .
$$

For the superposition of two linear wave components, Dalzell [12] expressed the combined linear and second-order potential function as:

$$
\Phi=\Phi^{(1)}+\Phi^{(2)}
$$

where the first-order velocity potential is:

$$
\Phi^{(1)}=\sum_{j=1}^{2} a_{j} \frac{g}{\omega_{j}} \frac{\cosh \left[k_{j}(z+h)\right]}{\cosh \left(k_{j} h\right)} \sin \psi_{j}
$$

and the second-order velocity potential is:

$$
\begin{gathered}
\Phi^{(2)}=\sum_{j=1}^{2} a_{j}^{2} \frac{3 \omega_{j}}{8} \frac{\cosh \left[2 k_{j}(z+h)\right]}{\sinh ^{4}\left(k_{j} h\right)} \sin \left(2 \psi_{j}\right)+ \\
a_{1} a_{2} A^{+}\left(k_{1}, k_{2}\right) \frac{\cosh \left[\left(k_{1}+k_{2}\right)(z+h)\right]}{\cosh \left[\left(k_{1}+k_{2}\right) h\right]} \sin \left(\psi_{1}+\psi_{2}\right)+ \\
a_{1} a_{2} A^{-}\left(k_{1}, k_{2}\right) \frac{\cosh \left[\left|k_{1}-k_{2}\right|(z+h)\right]}{\cosh \left[\left|k_{1}-k_{2}\right| h\right]} \sin \left(\psi_{1}-\psi_{2}\right)
\end{gathered}
$$

in which the choice of distribution of wave elevation does not affect the results of the wave-wave interaction coefficients. The sum frequency potential interaction coefficient $A^{+}$and difference frequency potential interaction coefficient $A^{-}$are as follows:

$$
\begin{gathered}
A^{+}\left(k_{1}, k_{2}\right)=-\frac{\omega_{1} \omega_{2}\left(\omega_{1}+\omega_{2}\right)}{D_{12}^{+}\left(k_{1}, k_{2}\right)}\left[1-\frac{\cos \left(\theta_{1}-\theta_{2}\right)}{\tanh \left(k_{1} h\right) \tanh \left(k_{2} h\right)}\right]+ \\
\frac{1}{2 D_{12}^{+}\left(k_{1}, k_{2}\right)}\left[\frac{\omega_{1}^{3}}{\sinh ^{2}\left(k_{1} h\right)}+\frac{\omega_{2}^{3}}{\sinh ^{2}\left(k_{2} h\right)}\right],
\end{gathered}
$$

and 


$$
\begin{gathered}
A^{-}\left(k_{1}, k_{2}\right)=\frac{\omega_{1} \omega_{2}\left(\omega_{1}-\omega_{2}\right)}{D_{12}^{-}\left(k_{1}, k_{2}\right)}\left[1+\frac{\cos \left(\theta_{1}-\theta_{2}\right)}{\tanh \left(k_{1} h\right) \tanh \left(k_{2} h\right)}\right]+ \\
\frac{1}{2 D_{12}^{-}\left(k_{1}, k_{2}\right)}\left[\frac{\omega_{1}^{3}}{\sinh ^{2}\left(k_{1} h\right)}-\frac{\omega_{2}^{3}}{\sinh ^{2}\left(k_{2} h\right)}\right]
\end{gathered}
$$

where the functions, $D_{12}^{+}\left(k_{1}, k_{2}\right)$ and $D_{12}^{-}\left(k_{1}, k_{2}\right)$ are defined as:

$$
D_{12}^{+}\left(k_{1}, k_{2}\right)=\left(\omega_{1}+\omega_{2}\right)^{2}-g\left(k_{1}+k_{2}\right) \tanh \left(\left(k_{1}+k_{2}\right) h\right),
$$

and

$$
D_{12}^{-}\left(k_{1}, k_{2}\right)=\left(\omega_{1}-\omega_{2}\right)^{2}-g\left|k_{1}-k_{2}\right| \tanh \left(\left|k_{1}-k_{2}\right| h\right) .
$$

The positive interaction terms given by eqn. (16) occur at the sum of the frequencies of the interacting wave components, and produce the sharpening of the free surface elevation maxima and flattening of the free surface elevation minima. The negative interaction terms given by eqn. (17) occur at the difference of the frequencies of the first-order wave components, and give the set-down of the water level under the wave group (More details are given by Dean and Dalrymple [1] and Chen [13]). After substituting this limit into the corresponding part of eqn. (11), and setting $a_{2}=a_{1}, \psi_{2}=\psi_{1}, \omega_{2}=\omega_{1}, k_{2}=k_{1}$ in eqn. (11) the unidirectional progressive wave potential, and wave elevation can be expressed as:

$$
\begin{gathered}
\Phi_{u n i}=\left(2 a_{1}\right) \frac{g}{\omega_{1}} \frac{\cosh \left[k_{1}(z+h)\right]}{\cosh \left(k_{1} h\right)} \sin \psi_{1}+ \\
\left(2 a_{1}\right)^{2} \frac{3 \omega_{1}}{8} \frac{\cosh \left[2 k_{1}(z+h)\right]}{\sinh ^{4}\left(k_{1} h\right)} \sin \left(2 \psi_{1}\right),
\end{gathered}
$$

and

$$
\begin{gathered}
\eta_{\text {uni }}=\left(2 a_{1}\right) \cos \psi_{1}-\frac{\left(2 a_{1}\right)^{2} k_{1}}{2 \sinh \left(2 k_{1} h\right)}+ \\
\frac{4 a_{1}^{2} k_{1}}{4 \tanh \left(k_{1} h\right)}\left[2+\frac{3}{\sinh ^{2}\left(k_{1} h\right)}\right] \cos \left(2 \psi_{1}\right)
\end{gathered}
$$

Eqn. (18) corresponds to a single second-order Stokes wave of amplitude $2 a_{1}$, following the non-zero mean elevation convention (see Mei [2]). These results can be checked by setting $a_{2}=a_{1}, \psi_{2}=\psi_{1}=0, \omega_{2}=\omega_{1}, k_{2}=k_{1}$ in eqns. (11) and (18) for the 
superposition of two waves which propagate in opposite directions (which should produce a single, second-order standing wave).

\section{Wave spectrum}

In Chen [13], by comparing wave simulations based on Bretschneider and JONSWAP spectra, it has been shown that extreme crest elevations are most likely to occur in a narrow-banded, long crested sea-state than in a broad-banded, shorted-crested sea-state. Consequently, a unidirectional Bretschneider spectrum is selected to represent the linear sea surface as follows:

$$
S(\omega)=\frac{5 E_{\omega}}{\omega_{P}}\left(\frac{\omega_{P}}{\omega}\right)^{5} e^{-\frac{5}{4}\left(\frac{\omega_{P}}{\omega}\right)^{4}}
$$

in which $S(\omega)$ represents the energy density at angular frequency $\omega, \omega_{P}$ denotes the angular frequency of peak energy and $E_{\omega}$ is the total energy.

Figure 2 presents the discrete spectrum, in which each spectral component incorporates the energy over a frequency range of $\pm \frac{1}{2} \Delta \omega$ either side of the frequency of interest, where $\Delta \omega=2 \pi /(m \Delta t)=0.0628 \mathrm{rad} / \mathrm{s}$. The first-order, Dalzell [12]'s theoretical solutions to second-order, and second-order sum (see Chen [13]), component time histories of the free surface elevation corresponding to this spectrum are illustrated in Figure 3. The results indicate that the predictions of the maximum crest elevation based on the fully nonlinear formulation are considerably larger than would be obtained from linear wave theory. Additionally, the theoretical linear solution is almost independent of the spreading effect. However, the second and higher order wave-wave interactions are affected by spreading. It is therefore useful to compare the unidirectional second-order sum frequency free surface elevation components with those for varying angles of spread.

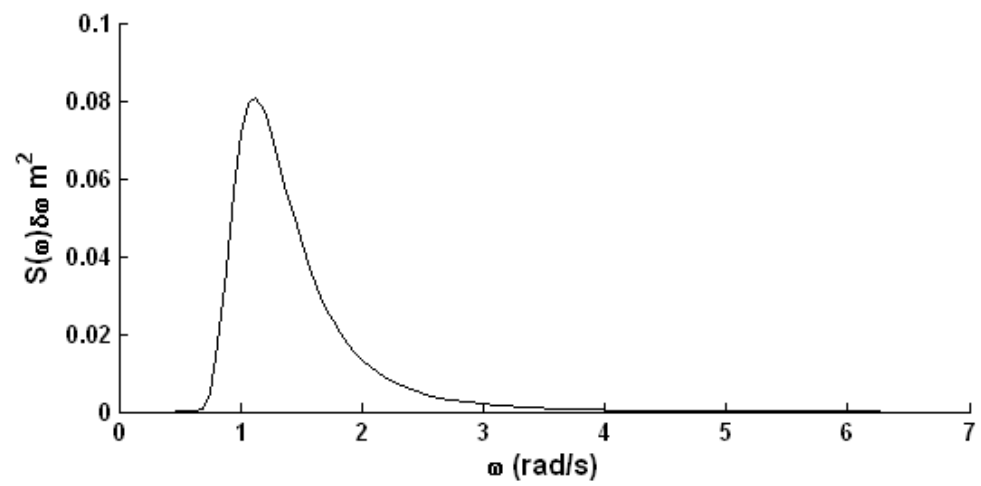

Figure 2: $\quad$ Unidirectional Bretschneider spectrum with $\omega_{P} \approx 1.177(\mathrm{rad} / \mathrm{s})$. 


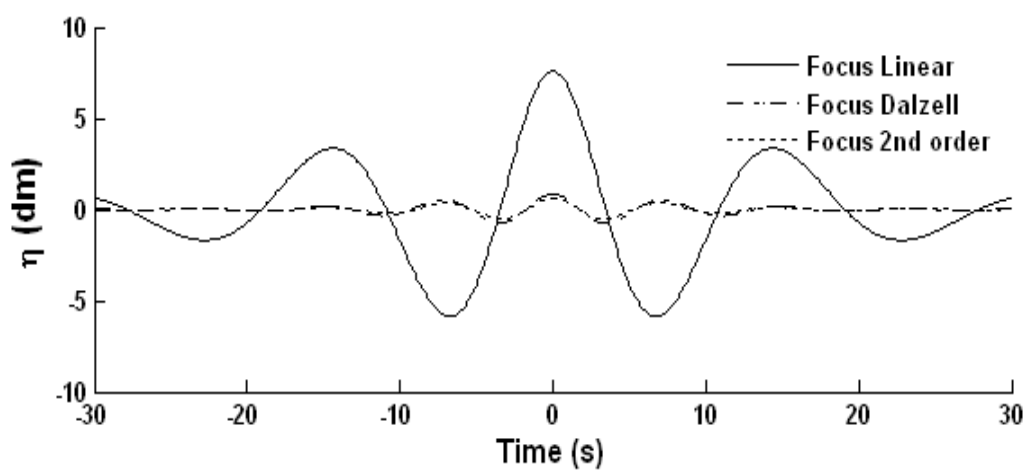

Figure 3: Comparison between time histories of the linear and nonlinear contribution.

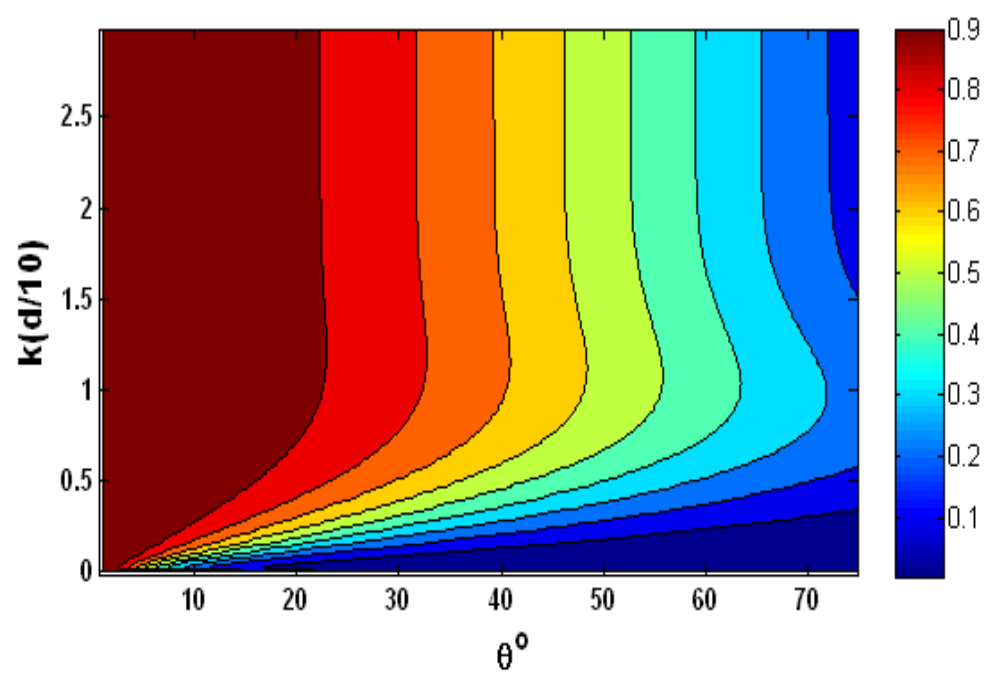

Figure 4: Directional spreading effects on the second-order coefficient with various $k d$ values.

Figure 4 shows that the resulting wave pattern due to the interaction of the two linear wave components is highly spread (for orange upwards) in shallow water where $k d$ is $<0.3$ and the depth influences the propagation directions of local waves. Moreover, Figure 5 demonstrates that the relative standard deviation of the second-order sum terms diminishes monotonically as a function of increasing angle of directional spreading. The relationship appears to be nearly parabolic in form. These results also imply the reduction in amplitude of the sum second-order free surface wave components. 


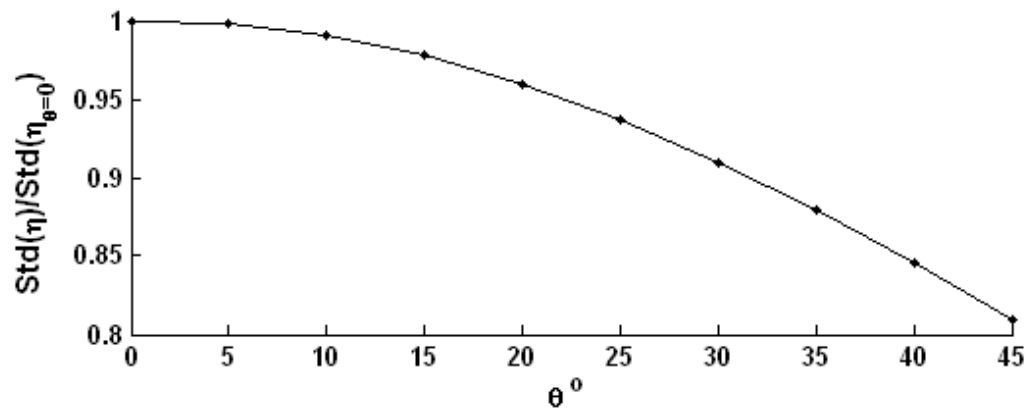

Figure 5: Estimated second-order coefficient with directional spreading effects.

\section{Wave-structure interaction in a directional spread sea-state}

It should be noted that only a small portion of the computational results are presented in this section. The free surface motion resulting from directional spreading of a selected incident second-order (sum) NewWave event associated with isolated platform loading column is predicted using the interaction theory represented in Linton and Evans [8]. As shown in Figure 6, the amplitude of the second-order sum free surface elevation components decreases as the spreading angle increases, which is consistent with the results discussed above.

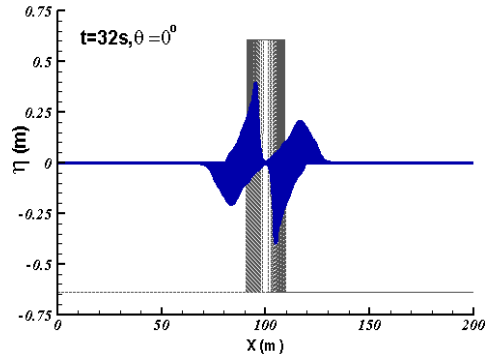

(a)

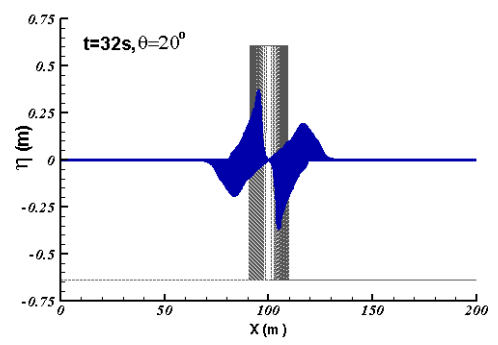

(c)

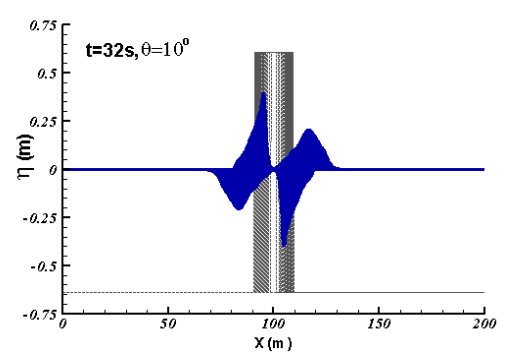

(b)

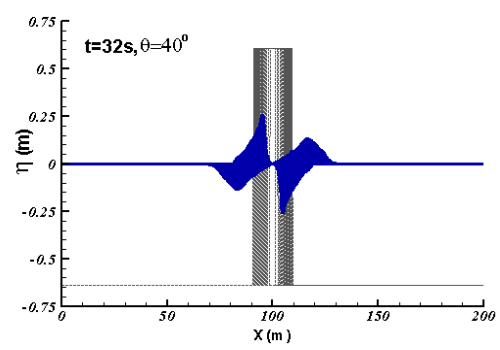

(d)

Figure 6: Free surface motion for a NewWave focused at $t=100 \mathrm{~s}$ with an isolated circular loading column of the platform. 


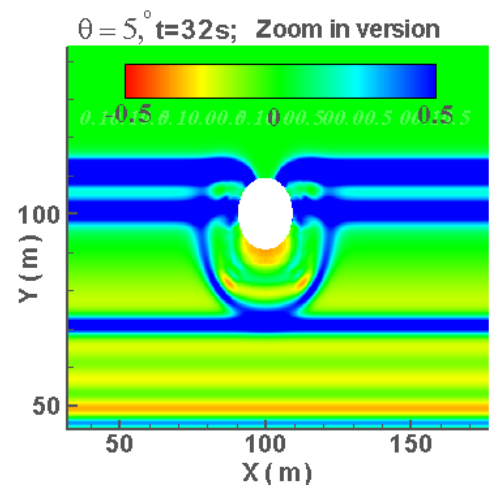

(a)

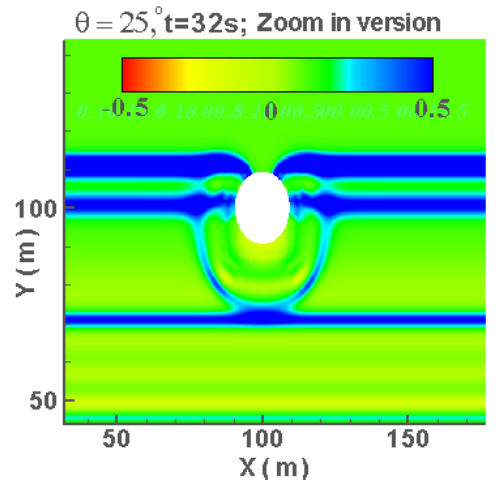

(c)

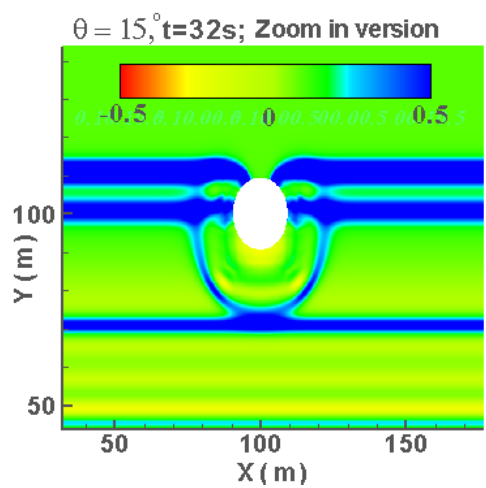

(b)

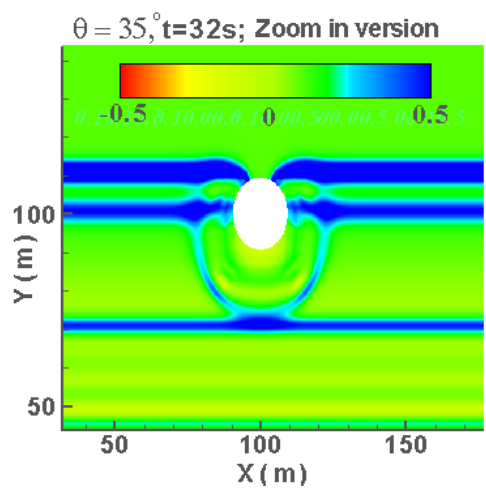

(d)

Figure 7: Directional spreading effects on focused NewWave-platform interactions at $t=32 s$.

Figure 7 further confirms the effects of the directional spreading by simulating a limited number of the extreme wave components for a Bretschneider spectrum. The results showed that the full second-order predictions of maximum wave loadings and their patterns relating to the interactions between the second-order wave components and a platform column were smaller than predictions based on unidirectional approximate second order theory. As expected, the magnitudes of the exact second-order free surface elevations reduced with increasing directional spreading angle. In contrast, this full nonlinear simulation displayed that the shape profile of the almost exact solution remained constant, even when the directional spreading angles were altered.

\section{Conclusions}

Using the NewWave formulation associated with a Bretschneider spectrum, the parabolic form of the second-order coefficient consists of the directional feature, 
and the interaction theory of Linton and Evans [8], a more realistic maximum free surface elevation and wave-structure interaction phenomenon has been predicted for a given directional spread sea-state. The exact second-order solution is affected by the directional spreading angle. The magnitude of the total wave free surface motion is found to be weakly dependent on the directional spreading angle.

\section{Acknowledgements}

The author would like to thank Professors A.G.L. Borthwick and P.H. Taylor at University of Oxford for their valuable advices to this work. The author is also very appreciative of unwavering support and excellent supervision of Dr D. Liang at University of Cambridge. The author is very grateful at being able to share his thoughts and ideas with these figures that have such high expertise in the field of Engineering.

\section{References}

[1] Dean, R. G., and Dalrymple, R. A. Water wave mechanics for engineers and scientist. World Scientific, Singapore. pp 41-69, 187-207, 1991

[2] Mei, C. C. The Applied Dynamics of Ocean Surface Waves. World Scientific, Singapore, 1989.

[3] Mei, C. C. Numerical methods in water-wave diffraction and radiation. Ann. Rev. Fluid Mech. 10:393-416, 1978.

[4] Ursell, F. Trapping modes in the theory of surface waves. Camb. Phil. Soc. 47, pp 347-358, 1951.

[5] Longuet-Higgins, M. S. Resonant interaction between two trails of gravity waves. J. Fluid Mech., 12: 1321-32, 1962.

[6] Lindgren, G. Some properties of a normal process near a local maximum, Ann. Math. Statist 41. (1870-1883), 1970.

[7] Boccotti, P. Some new results on statistical properties of wind waves. Applied Ocean Research 5(3) pp. 134-140, 1983.

[8] Linton, C. M., and Evans, D. V. The interaction of waves with arrays of vertical circular cylinders. J. Fluid Mech. Vol. 215, pp549-569, 1990.

[9] Tromans, P. S., and Anaturk, A. R. A new model for the kinematics of large ocean waves: Application as a design wave. ISOPE, Volume 3, Edinburgh, UK pp. 64-71, 1991.

[10] Jonathan, P., and Taylor, P. H. On irregular non-linear waves in a spread sea. J Offshore Mech Arctic Eng 119:37-41, 1997.

[11] Forristall, G. Z. Wave crest distributions: Observations and second-order theory. Journal of Phys. Oceanography, 30: 1931-1943, 1999.

[12] Dalzell, J. F. A note on finite depth second-order wave-wave interaction. Applied Ocean Research 21; pp.105-11, 1999.

[13] Chen, M. Wave statistics in directional spread sea-states and wave-wave interactions. M.Sc. thesis, University of Oxford, UK, 2008. 